\title{
Luminosity optimization near the beam-beam limit by increasing bunch length or crossing angle
}

\author{
F. Ruggiero and F. Zimmermann \\ CERN, SL Division, AP Group, CH-1211 Geneva 23, Switzerland
}

(Received 21 February 2002; published 18 June 2002)

\begin{abstract}
We discuss the choice of bunch length and crossing angle near the beam-beam limit in a storage-ring collider. First, we derive expressions for the tune shifts of either bunched or continuous round beams which are induced by a single collision with arbitrary crossing angle and bunch length and for the associated luminosities. Then, considering two collision points with alternating planes of crossing, we demonstrate that, if the total beam-beam tune shift is held constant, the collider luminosity increases as a function of bunch length and crossing angle. This implies a corresponding increase in the bunch intensity. As an illustration, we present numerical examples for a Large Hadron Collider upgrade and for the Very Large Hadron Collider.
\end{abstract}

DOI: 10.1103/PhysRevSTAB.5.061001

\section{INTRODUCTION}

Beam-beam tune shifts [1,2] and luminosity [3] for coasting beams colliding under a small crossing angle have been derived in the early days of the CERN intersecting storage rings. Most of the analyses at that time considered round beams, but included the variation of the beta functions around the collision point. The luminosity for the collision of beams of finite length with zero crossing angle was computed during the same period at SLAC [4], and, including a nonzero crossing angle, at LBL [5]. Luminosity formulas for a variety of cases were compiled in Ref. [6]. A compact expression for the zero crossing angle can be found in Ref. [7]. The report by Montague [2] extended the treatment of the beam-beam tune shift also to flat beams and nonzero dispersion. The effect of a finite bunch length on the beam-beam interaction of round beams was studied by Krishnagopal and Siemann in Ref. [8]. They found that due to the finite bunch length the Fourier coefficients in the beam-beam Hamiltonian are suppressed by a Gaussian form factor. During the design of the B factories, it was pointed out by Hirata that a large crossing angle can be beneficial for the collision of bunched electron-positron beams [9]. Even more recently, the collision of long proton "superbunches" (much longer than $\beta_{x, y}^{*}$ ) with a large crossing angle was suggested as a means to attain higher luminosity in future hadron colliders [10].

In this paper, we first derive a general expression for the tune shift of round beams of arbitrary bunch length colliding under an arbitrary crossing angle in a single collision point. In the limit of small crossing angles and for coasting beams, the formula obtained reproduces the earlier results [1,2]. We then present an expression for the total beam-beam tune shift for a colliding-beam storage ring with two interaction points (IPs) in which the (round) beams are crossed horizontally and vertically, respectively. We next derive expressions for the luminosity, again considering an arbitrary bunch length and crossing angle. Comparing the parameter dependence of the total tune shift with that for the luminosity, we conclude that, if
PACS numbers: 29.27.Bd

a collider operates near the beam-beam limit, the luminosity can be raised by a simultaneous increase in the product of the crossing angle and bunch length, and in the bunch intensity [see Eqs. (21) and (29)], while it is independent of the emittance. In the appendix, we describe a recipe for computing the tune shift for a particle at an arbitrary betatron amplitude and the associated tune footprint in the tune diagram, for both bunched and continuous beams. Although we do not explicitly consider parasitic beam-beam encounters (for bunched beams), their effect could be computed as well using the formalism presented here.

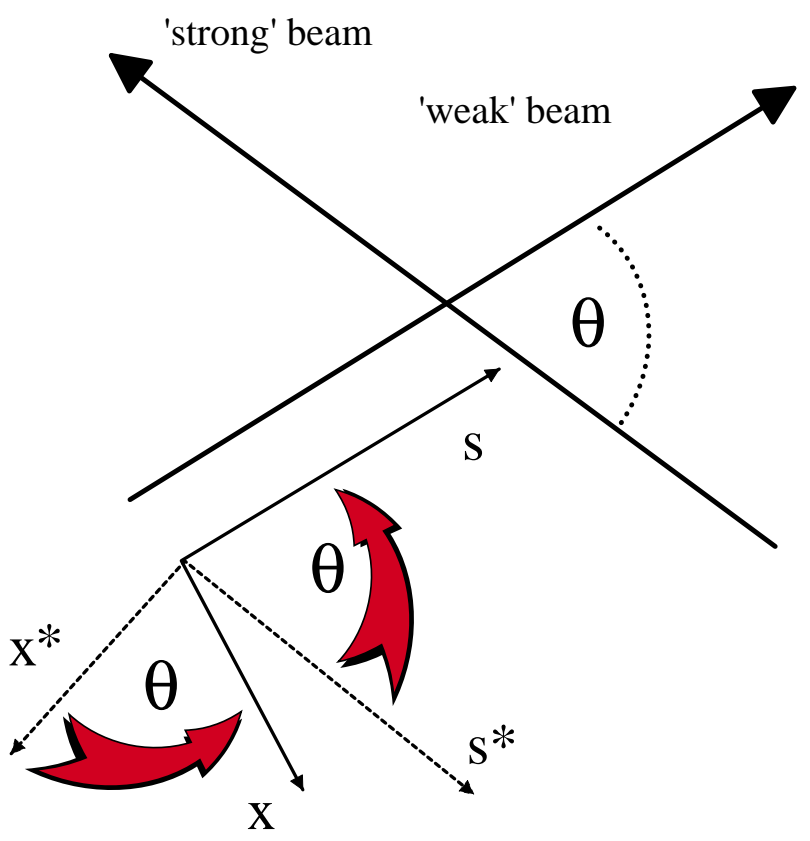

FIG. 1. (Color) Schematic of coordinate system for two beams colliding under a crossing angle $\theta$. The $s^{*}$ coordinate with asterisk is aligned with the axis of the "strong" beam, but points opposite to its direction of motion. The $s$ coordinate without asterisk refers to the frame of the "weak" beam for which the tune shift is to be calculated. The two frames are related via a rotation in the $s-x$ plane by the angle $\theta$. The vertical $(y)$ axis is perpendicular to the plane drawn and identical in the two coordinate systems. 
We illustrate our discussion by numerical examples for possible upgrade scenarios of the Large Hadron Collider (LHC) [11], presently under construction at CERN, and for the Very Large Hadron Collider (VLHC) [12], proposed in the U.S.

In the following, we use the coordinate system depicted in Fig. 1. The coordinates with the asterisk refer to the frame aligned in the direction of the "strong" beam, those without the asterisk to the reference frame of the "weak" beam, for which the tune shift is to be calculated. Throughout this paper, we consider ultrarelativistic beams whose energy is much larger than their rest mass.

\section{TUNE SHIFT FOR A SINGLE INTERACTION OF TWO ROUND BEAMS}

We first study the case of continuous beams. Without a crossing angle, the coordinate frames aligned with the two beams are identical. In this case, and for a round proton beam with Gaussian distribution in the radial direction, the force, e.g., in the strong-beam coordinate frame, easily follows from Maxwell's equations. Namely, a particle experiences the acceleration

$$
\begin{aligned}
& \frac{\partial^{2} x^{*}}{\partial s^{* 2}}=\frac{4 \lambda r_{p}}{\gamma} \frac{x^{*}}{r^{* 2}}\left[1-\exp \left(-\frac{r^{* 2}}{2 \sigma(s)^{2}}\right)\right], \\
& \frac{\partial^{2} y^{*}}{\partial s^{* 2}}=\frac{4 \lambda r_{p}}{\gamma} \frac{y^{*}}{r^{* 2}}\left[1-\exp \left(-\frac{r^{* 2}}{2 \sigma(s)^{2}}\right)\right],
\end{aligned}
$$

where $r^{* 2}=x^{* 2}+y^{* 2}, x^{*}$ and $y^{*}$ are the horizontal and vertical coordinates with respect to the beam center, $\lambda$ denotes the line density of the strong beam in units of $\mathrm{m}^{-1}$, $r_{p}$ is the classical proton radius [for other particle species of mass $M$ and charge $Q$, the corresponding radius $r_{M} \equiv$ $Q^{2} /\left(4 \pi \epsilon_{0} M c^{2}\right)$ should be taken], and $\sigma(s) \equiv \sigma_{x}(s)=$ $\sigma_{y}(s)$ is the transverse rms beam size, which varies with the longitudinal position $s$.

If the colliding beams were flat instead of round, Eqs. (1) and (2) would need to be replaced by more complicated expressions involving the complex error functions and the two rms transverse beam sizes [13]. This would add considerable complexity to the calculation and, more importantly, it would prevent a simplification and cancellation between the two planes which appears essential to our scheme of raising the luminosity. In the following, we will consider only the collision of round beams, which is described by Eqs. (1) and (2).

In the case with the crossing angle, say in the horizontal plane, we must apply a coordinate transformation to the weak-beam frame (without the asterisk):

$$
\begin{aligned}
& x^{*}=x \cos \theta-s \sin \theta, \\
& s^{*}=s \cos \theta+x \sin \theta .
\end{aligned}
$$

Figure 1 shows a schematic of the two coordinate systems. The associated transformations of the electric and magnetic fields are

$$
\begin{aligned}
E_{y}=E_{y}^{*} \quad \text { and } \quad E_{x}=E_{x}^{*} \cos \theta, \\
B_{y}=B_{y}^{*} \quad \text { and } B_{x}=B_{x}^{*} \cos \theta, \\
F_{x}=e E_{x}+c e B_{y} \quad \text { and } F_{y}=e E_{y}-c e B_{x},
\end{aligned}
$$

and, thus, the forces acting on a particle in the weak beam are

$$
F_{x}=e E_{x}^{*}(1+\cos \theta)=F_{x}^{*} \frac{1+\cos \theta}{2},
$$

and

$$
F_{y}=e E_{y}^{*}(1+\cos \theta)=F_{y}^{*} \frac{1+\cos \theta}{2} .
$$

For ease of notation we define the coefficient

$$
K \equiv \frac{4 \lambda r_{p}(1+\cos \theta)}{2 \gamma},
$$

and abbreviate the trigonometric expressions as $C \equiv \cos \theta$ and $S \equiv \sin \theta$. The coefficient $K$ of Eq. (10) is positive if the two colliding beams have equal sign of charge, as in the LHC. Otherwise the value of $\lambda$ should be chosen with a negative sign.

Using Eqs. (1), (2), (8), and (9), we can now write the acceleration of a particle in the weak beam as

$$
\begin{aligned}
f_{x} \equiv & \frac{\partial^{2} x}{\partial s^{2}}=K \frac{x C-s S}{(x C-s S)^{2}+y^{2}} \\
& \times\left[1-\exp \left(-\frac{(x C-s S)^{2}+y^{2}}{2 \sigma(s)^{2}}\right)\right], \\
f_{y} \equiv & \frac{\partial^{2} y}{\partial s^{2}}=K \frac{y}{(x C-s S)^{2}+y^{2}} \\
& \times\left[1-\exp \left(-\frac{(x C-s S)^{2}+y^{2}}{2 \sigma(s)^{2}}\right)\right] .
\end{aligned}
$$

The tune shift is obtained by integrating the transverse derivative of the force times the corresponding beta function over the longitudinal direction $s$, namely,

$$
\begin{aligned}
& \Delta Q_{x}=-\left.\frac{1}{4 \pi} \int_{-l / 2}^{l / 2} \frac{\partial f_{x}}{\partial x}\right|_{x=y=0} \beta_{x} d s, \\
& \Delta Q_{y}=-\left.\frac{1}{4 \pi} \int_{-l / 2}^{l / 2} \frac{\partial f_{y}}{\partial y}\right|_{x=y=0} \beta_{y} d s,
\end{aligned}
$$

where we assume that the beams are separated or shielded from each other at distances larger than $l / 2$ from the interaction point.

Computing the above derivatives yields

$$
\begin{aligned}
\left.\frac{\partial f_{x}}{\partial x}\right|_{x=y=0}=K C & \left\{-\frac{1}{s^{2} S^{2}}\left[1-\exp \left(-\frac{s^{2} S^{2}}{2 \sigma(s)^{2}}\right)\right]\right. \\
+ & \left.\frac{1}{\sigma(s)^{2}} \exp \left(-\frac{s^{2} S^{2}}{2 \sigma(s)^{2}}\right)\right\},
\end{aligned}
$$




$$
\left.\frac{\partial f_{y}}{\partial y}\right|_{x=y=0}=K\left\{\frac{1}{s^{2} S^{2}}\left[1-\exp \left(-\frac{s^{2} S^{2}}{2 \sigma(s)^{2}}\right)\right]\right\} .
$$

Inserting this into the preceding equations, and assuming $\beta(s)=\beta^{*}\left(1+s^{2} / \beta^{* 2}\right)$ where $\beta^{*} \equiv \beta_{x}^{*}=\beta_{y}^{*}$ denotes the beta function at the collision point (no quadrupole magnets over the length of the beam-beam interaction), we obtain the final expressions for the tune shift of two round coasting beams, or long superbunches, colliding under a horizontal angle $\theta$ :

$$
\begin{aligned}
\Delta Q_{x}= & +\frac{\lambda r_{p} \beta^{*}}{\pi \gamma}\left(\frac{1+\cos \theta}{2}\right) \cos \theta \int_{-l / 2}^{l / 2}\left(1+\frac{s^{2}}{\beta^{* 2}}\right) \\
& \times\left\{\frac{1}{s^{2} \sin ^{2} \theta}\left[1-\exp \left(-\frac{s^{2} \sin ^{2} \theta}{2 \sigma^{2}}\right)\right]\right. \\
& \left.-\exp \left(-\frac{s^{2} \sin ^{2} \theta}{2 \sigma^{2}}\right) \frac{1}{\sigma^{2}}\right\} d s \\
\Delta Q_{y}= & -\frac{\lambda r_{p} \beta^{*}}{\pi \gamma}\left(\frac{1+\cos \theta}{2}\right) \int_{-l / 2}^{l / 2}\left(1+\frac{s^{2}}{\beta^{* 2}}\right) \\
\times & \left\{\frac{1}{s^{2} \sin ^{2} \theta}\left[1-\exp \left(-\frac{s^{2} \sin ^{2} \theta}{2 \sigma^{2}}\right)\right]\right\} d s
\end{aligned}
$$

where $\sigma=\sigma(s)=\sigma^{*} \sqrt{1+s^{2} / \beta^{* 2}}, \quad \sigma^{*}=\sqrt{\epsilon \beta^{*}}=$ $\sqrt{\epsilon_{N} \beta^{*} / \gamma}$ using the geometric transverse emittance $\epsilon$ (taken to be equal in the horizontal and vertical planes) and the corresponding normalized emittance $\epsilon_{N}$, and, for a long superbunch of total length $l_{b}$ and bunch population $N_{b}$,

$$
\lambda \approx \frac{N_{b}}{l_{\text {bunch }}} \text { (superbunch). }
$$

For $\theta \ll 1$ the formulas (13) agree with those quoted by Keil in Ref. [3]. If we add the horizontal and vertical tune shifts, the first term in the expression for $\Delta Q_{x}$ almost cancels the full expression for $\Delta Q_{y}$, apart from a factor $(-\cos \theta)$. For a small crossing angle, the sum of the two tune shifts is determined by the last term in $\Delta Q_{x}$, which is proportional to $1 / \sigma(s)^{2}$. This cancellation between the terms describing the horizontal and vertical tune shifts, respectively, has an analog in the first order compensation of the linear tune shifts from (parasitic) long-range collisions for bunched beams, which occurs if at two (or more) interaction points the two beams are crossed alternatingly in the horizontal and vertical planes [14,15].
As an aside, in the case of flat-beam collisions it is difficult, if not impossible, to achieve a similar cancellation between two collision points, since, in general, the emittances, beta functions, and beam sizes are different in the two planes, and, in addition, the latter two vary differently with the longitudinal position $s$. For a related reason, the tune shifts of bunched flat beams which arise from long-range collisions differ by a factor $\kappa=\epsilon_{y} / \epsilon_{x}$ in the horizontal and vertical planes [16]. Therefore, the long-range tune shifts of flat beams cannot be compensated by alternating the plane of the beam crossing.

The expression for the tune shift in the case of bunched beams of Gaussian profile is similar to the previous result. However, we must take into account the fact that the density of the opposing beam now varies as

$$
G(s, x)=\exp \left(\frac{-(s+s C+x S)^{2}}{2 \sigma_{z}^{2}}\right) .
$$

As a consequence, both $f_{x}$ and $f_{y}$ must be multiplied with $G(s, x)$, and the expressions for $\partial f_{x} / \partial x$ and $\partial f_{y} / \partial y$ become

$$
\begin{aligned}
\left.\frac{\partial f_{x}}{\partial x}\right|_{x=y=0}=K & \left\{-\frac{C}{s^{2} S^{2}}\left[1-\exp \left(-\frac{s^{2} S^{2}}{2 \sigma(s)^{2}}\right)\right]\right. \\
& +\frac{C}{\sigma(s)^{2}} \exp \left(-\frac{s^{2} S^{2}}{2 \sigma(s)^{2}}\right)+\frac{(1+C)}{\sigma_{z}^{2}} \\
& \left.\times\left[1-\exp \left(-\frac{s^{2} S^{2}}{2 \sigma(s)^{2}}\right)\right]\right\} g(s)
\end{aligned}
$$

(Gaussian bunch),

$$
\left.\frac{\partial f_{y}}{\partial y}\right|_{x=y=0}=K\left\{\frac{1}{s^{2} S^{2}}\left[1-\exp \left(-\frac{s^{2} S^{2}}{2 \sigma(s)^{2}}\right)\right]\right\} g(s)
$$

(Gaussian bunch),

where $\left.g(s) \equiv G(s, x)\right|_{x=0}$ denotes the form factor

$$
g(s)=\exp \left(-\frac{s^{2}(1+\cos \theta)^{2}}{2 \sigma_{z}^{2}}\right) \text { (Gaussian bunch). }
$$

Note that now there is an additional term in the expression for $\partial f_{x} / \partial x$. The other difference to the coasting-beam case is that the field of the opposing beam is encountered only over a finite length and not over the full interaction region, which gives rise to the form factor $g(s)$. The expressions for the tune shift become

$$
\begin{aligned}
& \Delta Q_{x}=\frac{\lambda r_{p} \beta^{*}}{\pi \gamma}\left(\frac{1+\cos \theta}{2}\right) \int_{-l / 2}^{l / 2}\left(1+\frac{s^{2}}{\beta^{* 2}}\right)\left\{\frac{\cos \theta}{s^{2} \sin ^{2} \theta}\left[1-\exp \left(-\frac{s^{2} \sin ^{2} \theta}{2 \sigma^{2}}\right)\right]-\cos \theta \exp \left(-\frac{s^{2} \sin ^{2} \theta}{2 \sigma^{2}}\right) \frac{1}{\sigma^{2}}\right. \\
& \left.-\frac{1+\cos \theta}{\sigma_{z}^{2}}\left[1-\exp \left(-\frac{s^{2} \sin ^{2} \theta}{2 \sigma^{2}}\right)\right]\right\} g(s) d s \text { (Gaussian bunch), } \\
& \Delta Q_{y}=-\frac{\lambda r_{p} \beta^{*}}{\pi \gamma}\left(\frac{1+\cos \theta}{2}\right) \int_{-l / 2}^{l / 2}\left(1+\frac{s^{2}}{\beta^{* 2}}\right)\left\{\frac{1}{s^{2} \sin ^{2} \theta}\left[1-\exp \left(-\frac{s^{2} \sin ^{2} \theta}{2 \sigma^{2}}\right)\right]\right\} g(s) d s \text { (Gaussian bunch), }
\end{aligned}
$$


where $\lambda$ now denotes the peak line density, i.e., $\lambda=$ $N_{b} /\left(\sqrt{2 \pi} \sigma_{z}\right)$ for the Gaussian bunch, and $\sigma=\sigma(s)$. Again we observe the cancellation, up to a residual term proportional to $(1-\cos \theta)$, of the first term in the expression for $\Delta Q_{x}$ against the full expression for $\Delta Q_{y}$.

The effect of parasitic long-range encounters on the tune shift could be taken into account, by including the corresponding bunches in the form factor $g(s)$, so as to represent a series of bunches encountered at different longitudinal positions.

\section{TUNE SHIFT FOR TWO ALTERNATING CROSSINGS OF TWO ROUND BEAMS}

If there are two interaction points in the ring, and the beams cross one time under a horizontal crossing angle $\theta$, and the other time under a vertical angle $\theta$, the total tune shift $\Delta Q_{\text {tot }}$ is the same in both planes and simply given by the sum of $\Delta Q_{x}$ and $\Delta Q_{y}$ in Eq. (13) or Eq. (17), respectively. From (13) we obtain, for coasting beams or superbunches,

$$
\begin{aligned}
\Delta Q_{\mathrm{tot}}= & -\frac{\lambda r_{p} \beta^{*}}{\pi \gamma}\left(\frac{1+\cos \theta}{2}\right) \int_{-l / 2}^{l / 2}\left(1+\frac{s^{2}}{\beta^{* 2}}\right) \\
\times & \left\{(1-\cos \theta) \frac{1}{s^{2} \sin ^{2} \theta}\left[1-\exp \left(-\frac{s^{2} \sin ^{2} \theta}{2 \sigma^{2}}\right)\right]\right. \\
& \left.+\exp \left(-\frac{s^{2} \sin ^{2} \theta}{2 \sigma^{2}}\right) \frac{\cos \theta}{\sigma^{2}}\right\} d s \text { (superbunch), }
\end{aligned}
$$

which can also be rewritten as

$$
\begin{aligned}
& \Delta Q_{\mathrm{tot}}=-\frac{\lambda r_{p} \beta^{*}}{\pi \gamma}\left(\frac{1+\cos \theta}{2}\right) \frac{1}{\beta^{* 2} l \sigma^{*} \sin ^{3} \theta} \\
& \times\left[e^{-l^{2} \sin ^{2} \theta / 8 \sigma^{* 2}}\left\{-\left[4 \beta^{* 2}(\cos \theta-1)+l^{2} \cos \theta\right] \sigma^{*} \sin \theta\right\}+(\cos \theta-1)\left(4 \beta^{* 2}-l^{2}\right) \sigma^{*} \sin \theta\right. \\
& \left.+\sqrt{2 \pi} l\left[\beta^{* 2} \sin ^{2} \theta+(2 \cos \theta-1) \sigma^{* 2}\right] \operatorname{Erf}\left(\frac{l \sin \theta}{2 \sqrt{2} \sigma^{*}}\right)\right] \text { (superbunch), }
\end{aligned}
$$

where $\operatorname{Erf}(z)=2 \int_{0}^{z} e^{-t^{2}} d t / \sqrt{\pi}$ denotes the error function. The analogous expression for Gaussian bunches follows from (17),

$$
\begin{aligned}
\Delta Q_{\mathrm{tot}}= & -\frac{\lambda r_{p} \beta^{*}}{\pi \gamma}\left(\frac{1+\cos \theta}{2}\right) \int_{-l / 2}^{l / 2}(1-\cos \theta)\left(1+\frac{s^{2}}{\beta^{* 2}}\right) \\
& \times\left\{\frac{1}{s^{2} \sin ^{2} \theta}\left[1-\exp \left(-\frac{s^{2} \sin ^{2} \theta}{2 \sigma^{2}}\right)\right]+\cos \theta \exp \left(-\frac{s^{2} \sin ^{2} \theta}{2 \sigma^{2}}\right) \frac{1}{\sigma^{2}}\right. \\
& \left.+\frac{1+\cos \theta}{\sigma_{z}^{2}}\left[1-\exp \left(-\frac{s^{2} \sin ^{2} \theta}{2 \sigma^{2}}\right)\right]\right\} g(s) d s \text { (Gaussian bunch) }
\end{aligned}
$$

The integral can again be expressed in terms of error functions, but the result is more complex and we omit it here.

As an example, we consider the case of Gaussian bunches, with a small crossing angle (so that $\cos \theta \approx 1$; $\sin \theta \approx \theta$ ), and with a bunch length that is much shorter than the IP beta function $\beta^{*}$, but much larger than $\sigma^{*}$. In this case, formula (19) simplifies to

$$
\begin{gathered}
\Delta Q_{\mathrm{tot}} \approx-\frac{N_{b} r_{p} \beta^{*}}{2 \pi \gamma \sigma^{*} \sqrt{\sigma^{* 2}+\theta^{2} \sigma_{z}^{2} / 4}} \\
\left(\theta \ll 1, \sigma^{*} \ll \sigma_{z} \ll \beta^{*}, \text { Gaussian bunch }\right) .
\end{gathered}
$$

We recognize that the transverse beam size at the collision point $\sigma^{*} \equiv \sigma_{x, y}^{*}$, the bunch length $\sigma_{z}$, and the crossing angle $\theta$ enter in the combination $\left[\theta \sigma_{z} /\left(2 \sigma^{*}\right)\right]$, which is known as the Piwinski parameter. Indeed, the limiting case, Eq. (20), agrees with the result of Piwinski [17].

Using $\sigma^{*}=\sqrt{\epsilon \beta^{*} / \gamma}$, Eq. (20) can be rewritten in terms of the normalized emittance $\epsilon_{N}$ and "brilliance" $N_{b} / \epsilon_{N}$ as

$$
\begin{gathered}
\Delta Q_{\mathrm{tot}} \approx-\left(\frac{N_{b}}{\epsilon_{N}}\right) \frac{r_{p}}{2 \pi \sqrt{1+\left(\theta^{2} \sigma_{z}^{2} \gamma\right) /\left(4 \epsilon_{N} \beta^{*}\right)}} \\
\left(\theta \ll 1, \sigma^{*} \ll \sigma_{z} \ll \beta^{*}, \text { Gaussian bunch }\right) .
\end{gathered}
$$

We further note that for $\theta^{2} \sigma_{z}^{2} \gamma \gg 4 \beta^{*} \epsilon_{N}$ Eq. (20) simplifies to

$$
\begin{gathered}
\Delta Q_{\mathrm{tot}} \approx-\frac{N_{b} r_{p} \beta^{*}}{\pi \gamma \sigma^{*} \theta \sigma_{z}} \\
\left(\theta^{2} \sigma_{z}^{2} \gamma \gg 4 \beta^{*} \epsilon_{N}, \theta \ll 1, \sigma^{*} \ll \sigma_{z} \ll \beta^{*},\right.
\end{gathered}
$$

Gaussian bunch).

In the same limit, the formula for the luminosity [see Eq. (30)] shows exactly the same dependence on emittance, bunch length, and crossing angle. Thus, for a constant total beam-beam tune shift the luminosity can be raised only by increasing the bunch population or reducing $\beta^{*}$, with an implied simultaneous increase of bunch length or crossing angle. 


\section{LUMINOSITY FOR CONTINUOUS AND BUNCHED BEAMS}

For the luminosity only collisions occurring within a region, $-l_{\mathrm{det}} / 2<s<l_{\mathrm{det}} / 2$ are of interest where $l_{\mathrm{det}}$ denotes the effective length of the detector.

The luminosity is computed by convolving the two 3-dimensional distribution functions for the two colliding bunches or beams, $\rho_{1}$ and $\rho_{2}$, both in time and in space:

$$
\begin{aligned}
L= & 2 f_{\operatorname{coll}} c \cos ^{2}(\theta / 2) \int_{-l_{\mathrm{det}} / 2}^{l_{\mathrm{det}} / 2} d s \int_{-\infty}^{\infty} d x \int_{-\infty}^{\infty} d y \int_{-\infty}^{\infty} d t \\
& \times \rho_{1}(x, y, s-c t, s) \rho_{2}(x \cos \theta-s \sin \theta, y \\
& s \cos \theta+x \sin \theta+c t, s) .
\end{aligned}
$$

The factor $2 \cos ^{2}(\theta / 2)$ in front of the integral arises from the relative velocity of the two beams $[18,19]$. It is equal to $\sqrt{c^{2}\left(\overrightarrow{\boldsymbol{v}}_{1}-\overrightarrow{\boldsymbol{v}}_{2}\right)^{2}-\left(\overrightarrow{\boldsymbol{v}}_{1} \times \overrightarrow{\boldsymbol{v}}_{2}\right)^{2}} / c^{2}$, using $\overrightarrow{\boldsymbol{v}}_{1}=(0,0, c)$ and $\vec{v}_{2}=(-c \sin \theta, 0,-c \cos \theta)$. We here ignore the contribution to the relative velocity arising from the angular spread of the beam, which results in a negligible correction [5].

Consider first the case of round Gaussian bunches, for which $\rho_{1}$ and $\rho_{2}$ are given by the function

$$
\begin{aligned}
\rho_{i}(x, y, z, s)= & \frac{N_{b, i}}{(2 \pi)^{3 / 2} \sigma(s)^{2} \sigma_{z}} \\
& \times \exp \left(-\frac{x^{2}}{2 \sigma(s)^{2}}-\frac{y^{2}}{2 \sigma(s)^{2}}-\frac{z^{2}}{2 \sigma_{z}^{2}}\right)
\end{aligned}
$$

(Gaussian bunch),

where $i=1,2$. After integrating over $y, t$, and $x$ we obtain

$$
\begin{aligned}
L= & \frac{f_{\mathrm{coll}} N_{b, 1} N_{b, 2} \cos ^{2}(\theta / 2)}{(2 \pi)^{3 / 2} \sigma_{z}} \int_{-l_{\mathrm{det}} / 2}^{l_{\mathrm{det}} / 2} d s \\
& \times \frac{1}{\sigma(s)^{2}} \frac{1}{\sqrt{1+\cos ^{2} \theta+\frac{\sigma(s)^{2}}{2 \sigma_{z}^{2}} \sin ^{2} \theta}} \\
& \times \exp \left[-\frac{s^{2} \cos ^{2} \frac{\theta}{2}\left(1+\frac{\sigma(s)^{2}}{\sigma_{z}^{2}}-\left(1-\frac{\sigma(s)^{2}}{\sigma_{z}^{2}}\right) \cos \theta\right)}{\sigma(s)^{2}\left(1+\cos ^{2} \theta+\frac{\sigma(s)^{2}}{2 \sigma_{z}^{2}} \sin ^{2} \theta\right)}\right] .
\end{aligned}
$$

For a small crossing angle, $\theta \ll 1$, this simplifies to

$$
\begin{aligned}
& L= \frac{f_{\mathrm{coll}} N_{b, 1} N_{b, 2}}{(2 \pi)^{3 / 2} \sigma_{z}} \int_{-l_{\mathrm{det}} / 2}^{l_{\mathrm{det}} / 2} d s \frac{1}{\sigma(s)^{2}} \frac{1}{\sqrt{2+\theta^{2}\left(-1+\frac{\sigma(s)^{2}}{2 \sigma_{z}^{2}}\right)}} \\
& \times \exp \left[-\frac{s^{2} \frac{2 \sigma(s)^{2}}{\sigma_{z}^{2}}+\theta^{2} s^{2}\left(\frac{1}{2}-\frac{\sigma(s)^{2}}{4 \sigma_{z}^{2}}\right)}{2 \sigma(s)^{2}+\theta^{2} \sigma(s)^{2}\left(-1+\frac{\sigma(s)^{2}}{2 \sigma_{z}^{2}}\right)}\right] \\
&(\theta \ll 1) .
\end{aligned}
$$

Further considering the case of Gaussian bunches with an rms bunch length much shorter than the IP beta function (negligible hourglass effect) and the detector length, and an IP beam size much smaller than the bunch length, the expression of Eq. (26) becomes

$$
\begin{aligned}
& L \approx \frac{f_{\mathrm{coll}} N_{b 1} N_{b 2}}{4 \pi \sigma^{*} \sqrt{\sigma^{* 2}+\sigma_{z}^{2} \theta^{2} / 4}} \\
&(\theta \ll 1, \sigma_{z} \ll \beta^{*}, \sigma_{z} \ll l_{\mathrm{det}}, \\
&\left.\sigma^{*} \ll \sigma_{z}, \text { Gaussian }\right) .
\end{aligned}
$$

The luminosity reduction factor due to the crossing angle is the same as that for the beam-beam tune shift in Eq. (20). We can thus use Eq. (20) to reexpress the equation for the luminosity (27) in terms of the beam-beam tune shift. Assuming $N_{b} \equiv N_{b 1}=N_{b 2}$, we then obtain

$$
\begin{gathered}
L \approx \frac{\pi f_{\text {coll }} \gamma^{2} \sigma^{* 2}}{r_{p}^{2} \beta^{* 2}} \Delta Q_{\mathrm{tot}}^{2} \sqrt{1+\frac{\theta^{2} \sigma_{z}^{2}}{4 \sigma^{* 2}}} \\
\left(\theta \ll 1, \sigma_{z} \ll \beta^{*}, \sigma_{z} \ll l_{\mathrm{det}},\right. \\
\left.\sigma^{*} \ll \sigma_{z}, \text { Gaussian }\right),
\end{gathered}
$$

which shows that with constant beam-beam tune shift $\Delta Q_{\text {tot }}$, the luminosity increases for larger crossing angles and longer bunches. The constant beam-beam tune shift implies, e.g., that the bunch population is increased simultaneously, in proportion to the luminosity.

Long-range parasitic collisions may reduce the dynamic aperture and can also enhance the tune footprint at large betatron amplitudes. The complete tune footprint could be computed by including the parasitic bunches of the opposite beam in the density function $g(s)$ of Eq. (19). The strength of the long-range force increases for larger bunch populations, but it is reduced for a larger crossing angle. Therefore, if the parasitic collisions have a serious impact, for the luminosity optimization it might be advantageous to increase the crossing angle rather than the bunch length. More specifically, in order to ensure that the long-range effects are not aggravated, the crossing angle should be increased at least in proportion to the square root of the bunch population.

Equation (28) can be rewritten in terms of the normalized emittance $\epsilon_{N}$, brilliance $N_{b} / \epsilon_{N}$, and bunch population $N_{b}$,

$$
\begin{aligned}
L & \approx \frac{f_{\text {coll }} N_{b} \gamma}{4 \pi \beta^{*}}\left(\frac{N_{b}}{\epsilon_{N}}\right) \frac{1}{\sqrt{1+\left(\theta^{2} \sigma_{z}^{2} \gamma\right) /\left(4 \beta^{*} \epsilon_{N}\right)}} \\
& =\frac{f_{\text {coll }} \gamma}{2 r_{p}} \frac{N_{b}}{\beta^{*}}\left|\Delta Q_{\mathrm{tot}}\right| \\
(\theta & \left.\ll 1, \sigma_{z} \ll \beta^{*}, \sigma_{z} \ll l_{\mathrm{det}}, \sigma^{*} \ll \sigma_{z}, \text { Gaussian }\right),
\end{aligned}
$$

where, in the second line, we have expressed the luminosity in terms of the total beam-beam tune shift from two collision points, Eq. (21). Equation (29) shows that the luminosity at constant beam-beam tune shift $\left|\Delta Q_{\text {tot }}\right|$ is independent of the emittance and increases linearly with the bunch intensity. 
In the limit that $\theta^{2} \sigma_{z}^{2} \gamma \gg 4 \beta^{*} \epsilon_{N}$ (but still fulfilling all the previous conditions), the luminosity expression simplifies further, to

$$
\begin{gathered}
L \approx f_{\text {coll }} N_{b}^{2} /\left(2 \pi \theta \sigma_{z} \sigma^{*}\right) \\
\left(\theta^{2} \sigma_{z}^{2} \gamma \gg 4 \beta^{*} \epsilon_{N}, \theta \ll 1, \sigma_{z} \ll \beta^{*}, \sigma_{z} \ll l_{\text {det }},\right. \\
\left.\sigma^{*} \ll \sigma_{z}, \text { Gaussian }\right),
\end{gathered}
$$

which can be compared with the expression for the tune shift in Eq. (22).

For a superbunch, the calculation proceeds analogously, except that the density $\rho_{i}$ in Eq. (24) must be replaced by

$\rho_{i}(x, y, z, s)=\frac{N_{b, i}}{2 \pi \sigma(s)^{2} l_{\text {bunch }}} \exp \left(-\frac{x^{2}}{2 \sigma(s)^{2}}-\frac{y^{2}}{2 \sigma(s)^{2}}\right)$

(superbunch).

In this case, for a small crossing angle $(\theta \ll 1)$ and equal bunch population $\left(N_{b} \equiv N_{b, 1}=N_{b, 2}\right)$, the result after integration of the luminosity expression, Eq. (23), over $y, t$, and $x$ is [3]

$$
\begin{aligned}
L= & \frac{f_{\text {coll }} l_{\text {bunch }} \lambda \gamma}{2 \pi}\left(\frac{\lambda}{\epsilon_{N}}\right) \int_{-l_{\text {det }} /\left(2 \beta^{*}\right)}^{l_{\text {det }} /\left(2 \beta^{*}\right)} \frac{1}{1+u^{2}} \\
& \times \exp \left[-\frac{\beta^{* 2} \theta^{2}}{4 \sigma^{* 2}} \frac{u^{2}}{1+u^{2}}\right] d u \\
& (\theta \ll 1, \text { superbunch }),
\end{aligned}
$$

where we have assumed $\sigma(s)^{2}=\sigma^{* 2}\left(1+s^{2} / \beta^{* 2}\right)$. We recall that $\lambda=N_{b} / l_{\text {bunch. }}$.

\section{EXAMPLES: LHC UPGRADE AND VLHC}

The nominal LHC parameters [11] assume an rms bunch length $\sigma_{z}$ of $7.7 \mathrm{~cm}$, a crossing angle $\theta$ of $300 \mu \mathrm{rad}$, and a transverse rms spot size $\sigma^{*} \equiv \sigma_{x, y}^{*}$ of $16 \mu \mathrm{m}$. According to our above calculation we expect that the luminosity can be increased by increasing either the crossing angle or the bunch length, if we maintain a constant beam-beam tune shift by raising the bunch intensity.

We can compute the relative increase in luminosity $L$ and bunch population $N_{b}$ as a function of the product $\sigma_{z} \theta$ either using the approximations of Eqs. (20) and (28), which predict exactly the same dependence on $\sigma_{z}$ and $\theta$ for both $N_{b}$ and $L$, or we can alternatively evaluate the more accurate Eqs. (19) and (25), varying either the bunch length or the crossing angle, and always keeping the total beam-beam tune shift constant.

The result is shown in Fig. 2. Increasing the product $\left(\sigma_{z} \theta\right)$ by a factor of 10 yields an increase in the bunch population and in the luminosity by more than a factor of 5 .

The analogous calculation can be applied to the VLHC [12]. The beam-beam tune shift for the first stage of the VLHC is quite modest, about $\xi_{x, y} \approx 0.002$ for each of two

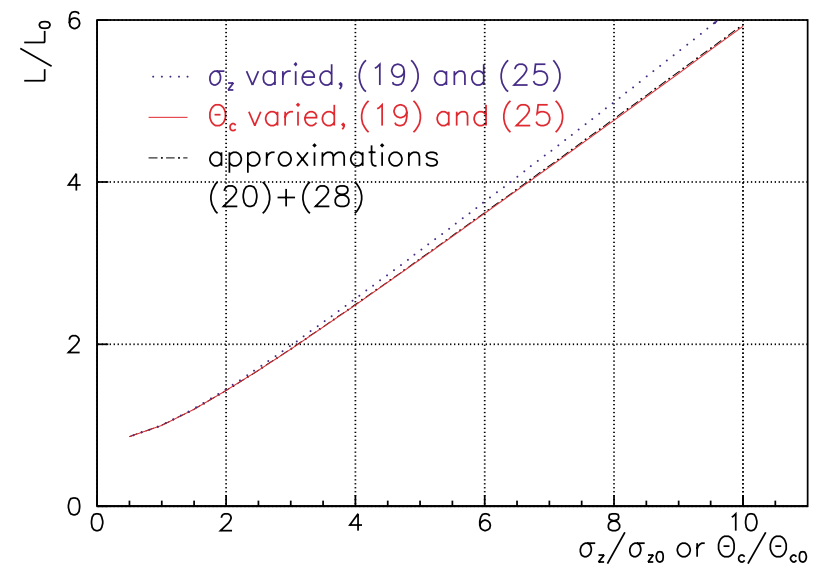

FIG. 2. (Color) Relative increase in LHC luminosity as a function of the relative increase of the product of rms bunch length and crossing angle, $\left(\sigma_{z} \theta\right)$, starting from a nominal bunch length $\sigma_{z}=7.7 \mathrm{~cm}$ and crossing angle $\theta=300 \mu \mathrm{rad}$ [11], and maintaining a constant total beam-beam tune shift for two collisions with alternating crossing. The transverse rms beam size is $\sigma^{*}=16 \mu \mathrm{m}$ and the interaction-point beta function $\beta^{*}=$ $0.5 \mathrm{~m}$. The subindex " 0 " refers to the nominal initial parameters listed above.

collision points. It is unlikely to limit the machine performance. The situation is different in the second stage of the VLHC, where the emittances will rapidly shrink due to radiation damping, and where a beam-beam tune shift of the order of 0.008 per IP may be reached after a few hours of collisions. We estimate that in the VLHC-II a quasistationary equilibrium between intrabeam scattering and radiation damping is reached when the rms transverse beam sizes at the collision point and the rms bunch length have decreased

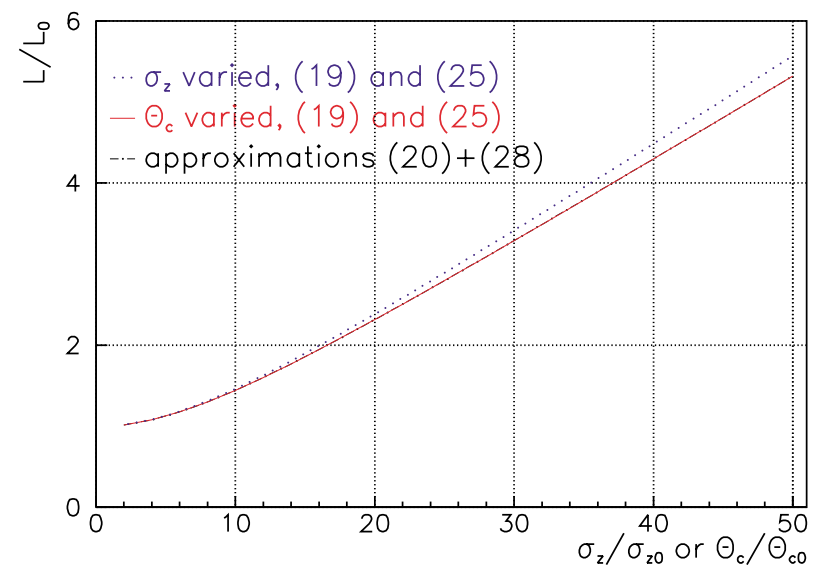

FIG. 3. (Color) Relative increase in VLHC-II luminosity as a function of the relative increase of the product of rms bunch length and crossing angle, $\sigma_{z} \theta$, starting from the quasiequilibrium state between radiation damping and intrabeam scattering [20,21] as estimated for the stage-II VLHC parameters [12], $\sigma_{z}=1.5 \mathrm{~cm}, \sigma^{*}=0.7 \mu \mathrm{m}, \beta^{*}=0.71 \mathrm{~m}$, and $\theta=10 \mu \mathrm{rad}$, assuming that $\sigma^{*}$ remains unchanged, and maintaining a constant total beam-beam tune shift for two collisions with alternating crossing. The subindex " 0 " refers to the initial parameters listed above. 
to about $0.7 \mu \mathrm{m}$ and $1.5 \mathrm{~cm}$, respectively [20,21]. Taking these numbers as the nominal values, and also assuming the VLHC design crossing angle of $10 \mu \mathrm{rad}$, we then compute the curves displayed in Fig. 3. Since the initial product of bunch length and crossing angle divided by the transverse rms beam size is smaller than for the LHC, a larger increase in bunch length or crossing angle is required, in order to obtain the same increase in luminosity. The nominal VLHC-II bunch population is small, only about $N_{b} \approx 7.5 \times 10^{9}$, and, hence, it can conceivably be raised, in proportion to the luminosity (vertical axis in the plot) so as to follow the curve in Fig. 3. Note that a longer bunch length also reduces the intrabeam scattering, an effect which has not been included in Fig. 3.

From our discussion it is clear that bunch length and crossing angle are important beam parameters whose control and adjustment during the store will not only render the operation of future colliders much more flexible, but may also substantially increase the integrated luminosity.

\section{CONCLUSION}

In this report, we have derived general analytical expressions for the beam-beam tune shift of round Gaussian bunches or superbunches of arbitrary length colliding under an arbitrary crossing angle and for the associated luminosities. The tune-shift formulas are strictly valid in the ultrarelativistic limit where the electromagnetic field is perpendicular to the direction of the beam propagation.

The expressions which we have obtained suggest that the luminosity of a collider can be raised by simultaneously increasing the bunch population and the product of bunch length and crossing angle, while maintaining a constant beam-beam tune shift. The increase in the crossing angle or bunch length differs from the conventional design approach.

We have illustrated our recipe by numerical examples for a possible LHC upgrade and for the VLHC, indicating potential gains in luminosity by a factor of 5 .

The corresponding formulas for the collision of flat beams would be more complicated than the round-beam equations presented in this report. For flat beams the total tune shifts are not significantly reduced by employing two collision points with alternating crossing, and, in particular, the expressions for the total tune shifts do not simplify in the same way as in the case of round beams. As a consequence, for flat beams the potential luminosity gains from increasing the crossing angle or the bunch length are likely to be smaller than those for round beams.

\section{ACKNOWLEDGEMENTS}

We thank W. Herr and H. Grote for helpful discussions and acknowledge constructive comments by K.-H. Schindl and R. Garoby.

\section{APPENDIX A: TUNE SHIFT WITH AMPLITUDE}

An alternative, more general approach of computing the beam-beam tune shift, including its dependence on the transverse amplitude, starts from the beam-beam potential in action-angle variables. In first order perturbation theory, the effective potential determining the tune shifts can be computed by integrating the local beam-beam potential over the longitudinal coordinate $s$. The local potential itself is obtained by integrating the beam-beam force in the radial direction.

Specifically, considering a particle with arbitrary longitudinal position, i.e., which collides with the center of the opposing beam at position $s_{0}$, the effective potential reads

$$
\begin{aligned}
& U\left(J_{x}, J_{y}, \phi_{x}, \phi_{y}, s_{0}, \theta\right) \\
& =-\frac{(1+\cos \theta) r_{p} \lambda}{\pi \gamma} \int_{-l / 2}^{l / 2} d s \\
& \quad \times G\left(s-s_{0}, x\right) \int_{-\infty}^{R(s)} \frac{d w}{w}\left[1-\exp \left(-\frac{w^{2}}{2}\right)\right],
\end{aligned}
$$

with

$$
\begin{aligned}
R(s) & \equiv\left(\frac{x \cos \theta-s \sin \theta)^{2}+y^{2}}{\epsilon \beta(s)}\right)^{1 / 2}, \\
x & =\sqrt{2 J_{x} \beta_{x}(s)} \cos \phi_{x}, \\
y & =\sqrt{2 J_{y} \beta_{y}(s)} \cos \phi_{y},
\end{aligned}
$$

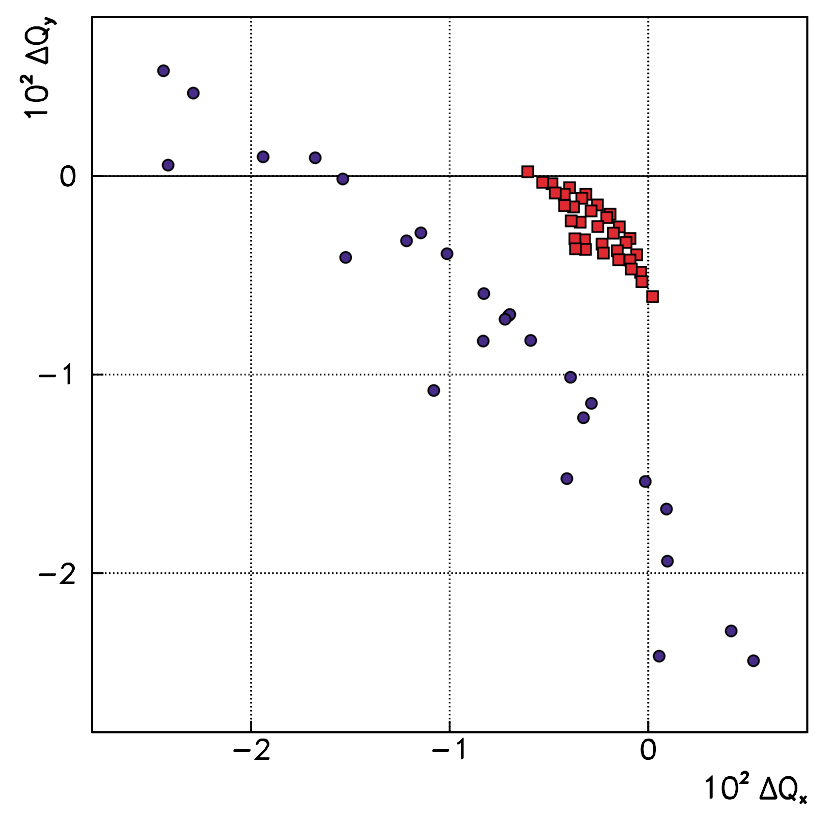

FIG. 4. (Color) Tune footprints for superbunches colliding under two different crossing angles: $\theta=400 \mu \mathrm{rad}$ (blue circles) and $\theta=1 \mathrm{mrad}$ (red squares), and betatron amplitudes extending from 0 to $6 \sigma$. Other parameters: line density $\lambda=$ $8.8 \times 10^{11} \mathrm{~m}^{-1}$, IP beta functions $\beta^{*} \equiv \beta_{x, y}^{*}=0.25 \mathrm{~m}$, total interaction length per IP $l=40 \mathrm{~m}$. 
where $\beta(s)=\beta^{*}\left(1+s^{2} / \beta^{* 2}\right), \epsilon$ is the transverse geometric emittance, and, for a Gaussian bunch, $G(s, x)$ was defined in Eq. (15), whereas for a superbunch $G(s, x)=1$. We have normalized the potential $U$ such that the azimuthal angle around the storage ring would be the associated time coordinate.

The tune shifts are now obtained by differentiating the effective potential $U$ with respect to the two action variables and averaging over the angles, i.e.,

$$
\Delta Q_{x, y}=\left\langle\frac{\partial U}{\partial J_{x, y}}\right\rangle_{\phi_{x}, \phi_{y}},
$$

where the angular brackets denote an average over the angle variables $\phi_{x}$ and $\phi_{y}$, namely,

$$
\langle(\cdots)\rangle \equiv \frac{1}{4 \pi^{2}} \int_{0}^{2 \pi} d \phi_{x} \int_{0}^{2 \pi} d \phi_{y}(\cdots) .
$$

Explicitly, for superbunches the derivatives entering in Eq. (A2) are

$$
\begin{gathered}
\frac{\partial U}{\partial J_{x}}=-\frac{(1+\cos \theta) r_{p} \lambda}{\pi \gamma} \int_{-l / 2}^{l / 2} d s\left\{\left[1-\exp \left(-\frac{R(s)^{2}}{2}\right)\right] \frac{\cos \theta(x \cos \theta-s \sin \theta) \cos \phi_{x}}{(x \cos \theta-s \sin \theta)^{2}+y^{2}} \sqrt{\frac{\beta(s)}{2 J_{x}}}\right\} \text { (superbunch), } \\
\frac{\partial U}{\partial J_{y}}=-\frac{(1+\cos \theta) r_{p} \lambda}{\pi \gamma} \int_{-l / 2}^{l / 2} d s\left\{\left[1-\exp \left(-\frac{R(s)^{2}}{2}\right)\right] \frac{y \cos \phi_{y}}{(x \cos \theta-s \sin \theta)^{2}+y^{2}} \sqrt{\frac{\beta(s)}{2 J_{y}}}\right\} \text { (superbunch). }
\end{gathered}
$$

Typical tune footprints obtained by solving Eq. (A2) numerically for a possible LHC upgrade using superbunches are shown in Fig. 4. In order to compute the tune shift of a particle at the head or tail of a superbunch, e.g., a particle which experiences the field of the other beam only between $-l / 2$ and $s_{\max }<l / 2$, the upper limits of integration in Eqs. (A4) and (A5) must be adjusted accordingly.

For Gaussian bunches, an additional term in $\partial U / \partial J_{x}$ arises from the derivative of $G\left(s-s_{0}, x\right)$ :

$$
\begin{aligned}
\frac{\partial U}{\partial J_{x}}=-\frac{(1+\cos \theta) r_{p} \lambda}{\pi \gamma} \int_{-l / 2}^{l / 2} d s & \left\{\left[1-\exp \left(-\frac{R(s)^{2}}{2}\right)\right] \frac{\cos \theta(x \cos \theta-s \sin \theta) \cos \phi_{x}}{(x \cos \theta-s \sin \theta)^{2}+y^{2}}\right. \\
& \times \sqrt{\frac{\beta(s)}{2 J_{x}}}-\sqrt{\frac{\beta_{x}(s)}{2 J_{x}}} \cos \phi_{x} \frac{\left(s-s_{0}\right) \sin \theta(1+\cos \theta)}{\sigma_{z}^{2}} \int_{-\infty}^{R(s)} \frac{d w}{w} \\
& \left.\times\left[1-\exp \left(-\frac{w^{2}}{2}\right)\right]\right\} G\left(s-s_{0}, x\right) \text { (Gaussian bunch), } \\
\frac{\partial U}{\partial J_{y}}=-\frac{(1+\cos \theta) r_{p} \lambda}{\pi \gamma} \int_{-l / 2}^{l / 2} d s\{[ & {\left[1-\exp \left(-\frac{R(s)^{2}}{2}\right)\right] } \\
& \left.\times \frac{y \cos \phi_{y}}{(x \cos \theta-s \sin \theta)^{2}+y^{2}} \sqrt{\frac{\beta(s)}{2 J_{y}}}\right\} G\left(s-s_{0}, x\right) \text { (Gaussian bunch). }
\end{aligned}
$$

[1] E. Keil, C. Pellegrini, and A. M. Sessler, CRISP 72-34, BNL Report No. 17017, 1972.

[2] B. W. Montague, CERN Report No. CERN/ISR-GS/75-36, 1975.

[3] E. Keil, Nucl. Instrum. Methods 113, 333 (1973).

[4] G. Fischer, SPEAR Note No. 154, 1972.

[5] L. Smith, PEP Note No. 20, 1972.

[6] T. Suzuki, KEK Report No. KEK-76-3, 1976.

[7] N. Dikanski and D. Pestrikov, The Physics of Intense Beams and Storage Rings (AIP, New York, 1994).

[8] S. Krishnagopal and R. Siemann, Phys. Rev. D 41, 2312 (1990).

[9] K. Hirata, Phys. Rev. Lett. 74, 2228 (1995).

[10] K. Takayama, J. Kishiro, M. Sakuda, and M. Wake (to be published).

[11] The LHC Study Group, Report No. CERN/AC/95-05, 1995. See also the project web page http://lhc.web. cern.ch/lhc

[12] The VLHC Design Study Group, Report No. FermilabTM-2149 2001. See also the project web page http:// www.vlhc.org
[13] M. Bassetti and G. A. Erskine, CERN Report No. CERNISR-TH/80-06, 1980.

[14] D. Neuffer and S. Peggs, SSC Report No. SSC-63, 1986.

[15] W. Herr, CERN Report No. CERN-SL-90-06, 1990 (AP).

[16] S. Peggs, in Proceedings of the Workshop on the Effect of Synchrotron Radiation in the VLHC, Brookhaven National Laboratory, 2000 (unpublished).

[17] A. Piwinski, DESY Report No. DESY 77/18, 1977.

[18] C. Moller and K. Danske, Vidensk. Selsk. Mat.-Fys. Medd. 23, 1 (1945).

[19] M. Furman and M. Zisman, in Handbook of Accelerator Physics and Engineering, edited by A.W. Chao and M. Tigner (World Scientific, Singapore, 1999).

[20] F. Zimmermann, in Proceedings of the XXIXth SLAC Summer Institute (SSI2001), SLAC, Stanford, 2001 (SLAC, Stanford, to be published).

[21] F. Zimmermann, in Proceedings of the 18th International Conference on High Energy Accelerators (HEACC2001), Tsukuba, Japan, 2001 (unpublished), http://wwweng.kek.jp/heacc2001/; CERN Report No. CERN-SL2001-009 AP, 2001. 